\title{
An investigation into patients' quality of life following intensive care and general surgical admissions
}

Robert Goddard, BSc (Hons) Physiotherapy, Senior I Physiotherapist, Darlington Memorial Hospital, UK

E-mail: Robert.Goddard@cddah.nhs.uk

Key words: general surgery intensive care physical function quality of life rehabilitation

\author{
SUMMARY \\ * This paper presents the findings of a study that aimed to \\ establish the quality of life of subjects following \\ intensive care and general surgery. \\ * Subjects attending a district general hospital over a \\ twelve-month period were contacted twelve weeks post \\ discharge. \\ * Data were collected using the SF-36 quality of life \\ questionnaire. \\ * Subjects admitted to intensive care for three nights or \\ more had a significantly reduced level of physical \\ function twelve weeks post discharge. \\ * Social Functioning and the Emotional Role were found \\ to be more greatly affected than other aspects of quality \\ of life in this sample. \\ * These findings demonstrate the need for holistic \\ rehabilitation following intensive care and general \\ surgical admissions.
}

\section{INTRODUCTION}

As a result of patients surviving more serious illnesses, symptoms such as generalised muscle weakness, neuropathy, lack of confidence and loss of functional activity are increasing following hospital discharge. Rehabilitation programmes are aimed at treating such symptoms. As these programmes develop there is a need to ensure that they are meeting the complex holistic needs of critical care survivors. This study explored the change in patients' quality of life following hospital admission to intensive care or for major general surgery.

Current research indicates that patients have significantly reduced functional ability following medical recovery from prolonged intensive care admissions and major surgery. This loss of physical function is despite their medical recovery and leads, in many cases, to a reduced quality of life following illness or surgery (Griffiths and Jones, 1999; McHugh et al., 1994; Pettila et al., 2000; Nielsen et al., 1997, Weinert et al., 1997; Heyland et al., 2000)

When assessing interventions in acute/critical care there is an increasing need to move beyond short-term survival to establish how survivors function and feel (Heyland and Kutsogiannis, 2000). There is also a need for a broad range of studies to assist in the assessment of patient outcomes following intensive care (Orlando, 2000). Hospital discharge is only a preliminary measure of therapeutic outcomes and longer-term measures account for the effects of hospitalisation on the patient's future quality of life (Jordan-Marsh, 2002).

In the UK, the Department of Health (2000) has debated the need for clinics following discharge from hospital for intensive care patients. This has then been supported by the NHS Modernisation Agency's (2003) review of critical care outreach services, which stated the need to implement these services as soon as possible. However a review by Lewis (2003) concluded that further research into the effects of rehabilitation on patients following intensive care unit (ICU) admission was required.

Against the background described above, the aims of this study were to:

* Establish subjects' quality of life twelve weeks following discharge from hospital

* Compare this to subjects' assessment of their pre-admission level of function

* Establish which aspects of quality of life were affected by hospitalisation

\section{METHODS}

\section{Quality of Life Measures}

The study aimed to assess the physical function and quality of life of subjects. Various quality of life measures were considered. A tool that has been used increasingly in critical care studies is the Short Form 36 (SF-36) Health Survey (Jordan-Marsh, 2002). This is a questionnaire which, when analysed, gives scores on different aspects of a subject's quality of life, including physical function. It has also been developed as a clinical tool for use in planning care (Ware et al., 2000). Further research into survivors of sepsis in the ICU concluded that the SF-36 demonstrated good reliability and validity when measuring health-related quality of life (Heyland et al., 2000). It has also been commonly used in research. (A litera- 
ture search using PubMed from 1998-2003 yielded 1816 references). As this tool was an appropriate measure, was common to research in the clinical area and had been shown to be reliable in similar research it was deemed to be appropriate for this study.

The most recent version of the SF-36 tool (version 2), which was used for this study, was developed in 2000 along with a range of norm-based scores derived from a large general population. In this study, one modification was made to the questionnaire: the third question, which was related to physical function, was repeated. This question gave subjects the opportunity to describe their level of physical function both pre-admission and twelve weeks following discharge; when the questionnaire was completed.

\section{Sample}

The Local Research Ethics Committee granted approval for this study. Prior to commencing the study permission to contact subjects was obtained from the clinical lead consultant within each of the hospital's directorates. Each subject's General Practitioner (GP) was also contacted prior to the survey being posted to ensure it was appropriate to include the subject in the study.

The sample of subjects was dictated by the clinical caseload of the participating hospital. All patients were in-patients at a district hospital serving a population of over 100,000 people.

Subjects were excluded from the study if they were terminally ill, or mentally or physically unable to complete the questionnaire. Subjects were also excluded if they were discharged outside of the geographical area of the hospital in which the study was based.

Subjects were recruited to the study by ward staff who completed referral forms. In order to reduce the potential for error the procedure was overseen by the researcher (RG) reviewing admissions to the surgical wards and the ICU. Data were collected over a twelvemonth period between January 1st 2002 and January 1st 2003.

The survey established pre-admission level of physical function and post-discharge quality of life and was sent to subjects who had undergone major general surgery or had been admitted to ICU for three nights or more. Major surgery was classified as those patients admitted under major surgery pathways of care in the hospital. Surgical procedures included hemicolectomy, nephrectomy, laparotomy (exploratory and emergency), anterior resection, sigmoid colectomy, abdominal aortic aneurysm repair, pyloroplasty, and gastrectomy. ICU patients were admitted to a general ICU with conditions including septicaemia, adult respiratory distress syndrome, multi-organ failure (including those following major surgery), anaphylaxis, and in one case multiple rib and sternal fractures.

Subjects were contacted by post twelve weeks following discharge from hospital and asked to complete the SF-36 survey. Return was requested by 'Freepost' within four weeks. A letter reminding subjects to complete the questionnaire was sent to each subject three weeks after it was posted. Subjects received a covering letter with the questionnaire explaining the aims of the study and how to complete the questionnaire. All questionnaires were coded anonymously, although coding enabled the questionnaires to be traced to either surgical or intensive care admissions.

All subjects received rehabilitation until safe discharge home was possible. There was no specific post critical care rehabilitation given to subjects following discharge. In-patient rehabilitation and advice included physiotherapy, occupational therapy, nursing and medical input.

In total 157 patients were referred for inclusion, of which 140 patients were contacted. The 17 subjects who were not contacted were excluded at their GP's request. A 44\% response rate was achieved $(n=61)$. Responses were from 14 subjects admitted to ICU of whom seven were male and seven were female. Their age ranged from 21-78 years (mean 60.9). A further 47 subjects who underwent major surgery responded. Of these, 28 were male and 19 were female, with an age range of $17-83$ years (mean 66.8).

\section{RESULTS}

\section{Data analysis}

Statistical comparisons were made between the two physical function scores (PF1 and PF2; see below) given by the subjects and also between the two sample groups. Using scoring systems developed by Ware et al. (2000) the SF-36 responses gave subjects scores in a variety of health related areas (see below). Comparing these scores was internally reliable, as the data had been generated through the same collection procedure.

\section{Limitations}

The benefits of the SF-36 are noted above. However, a limitation in the use of this version of the survey is that it uses norm-based values derived from an American population. (The most recent version using British norm-based scores dates back to 1996). For this reason no statistical analyses were made between norm-based scores and the subjects' responses.

\section{Results}

Data collected from the subjects were in the form of their responses to the SF-36 v2 questionnaire. It gives scores on a number of scales: Physical Function (PF), Role-Physical (RP), Bodily Pain (BP), General Health (GH), Vitality (VT), Social Functioning (SF), Role-Emotional (RE), Mental Health $(\mathrm{MH})$. Because question 3 of the questionnaire was repeated Physical Function was divided into PF1 and PF2. PF1 was the response to the question, 'How was your physical function before admission?' PF2 was the response to the question, 'How is your physical function now?' By splitting the question in this way it was possible to compare pre-admission and three-month post discharge physical function.

The SF-36 responses for the ICU sample and the surgery sample are illustrated below (Table 1). Table 1 shows mean scores and ranges for the ICU and surgery groups for each scale score on the SF-36 v2 Health Survey.

Table 1 demonstrates the large difference between PF1 and PF2 for the ICU group and in both groups SF, RP and RE are lower than other scores. This indicates that subjects perceived their social function and physical and emotional roles to be the most severely affected following their hospital admission.

The difference in PF scores is illustrated further in Table 2. There was a statistically significant difference in the deterioration of physical function in the ICU group from pre-admission to twelve weeks post discharge $(p=0.04, t=2.28 \mathrm{df}=13)$, whereas the surgery group had roughly the same functional level ( $p=n s$ ). 


\begin{tabular}{|c|c|c|c|c|c|c|c|c|c|}
\hline & PF 1 & PF 2 & $\mathrm{RP}$ & $\mathrm{BP}$ & $\mathrm{GH}$ & VT & SF & $\mathrm{RE}$ & $\mathrm{MH}$ \\
\hline & ICU & & & & & & & & \\
\hline $\mathrm{n}$ & 14 & 14 & 13 & 13 & 13 & 13 & 14 & 12 & 13 \\
\hline Mean & 38.24 & 28.57 & 31.11 & 41.51 & 38.03 & 38.88 & 29.19 & 25.11 & 38.31 \\
\hline Standard deviation & 16.08 & 13.16 & 11.21 & 10.84 & 14.91 & 11.34 & 14.42 & 15.68 & 14.89 \\
\hline \multirow[t]{2}{*}{ Range } & $15-57$ & $15-51$ & $18-57$ & $25-62$ & $16-62$ & $24-65$ & $13-57$ & $9-56$ & $16-64$ \\
\hline & Surge & & & & & & & & \\
\hline $\mathrm{n}$ & 47 & 47 & 46 & 47 & 47 & 47 & 47 & 45 & 47 \\
\hline Mean & 41.92 & 40.11 & 33.18 & 43.94 & 43.77 & 41.26 & 38.52 & 35.49 & 45.68 \\
\hline Standard deviation & 13.52 & 12.59 & 10.27 & 11.12 & 10.25 & 9.52 & 12.27 & 12.84 & 10.71 \\
\hline Range & $15-57$ & $15-57$ & $18-57$ & $25-62$ & $26-64$ & $21-61$ & $13-57$ & $9-56$ & $16-64$ \\
\hline
\end{tabular}

Table 1. SF-36 v2 Scale scores between groups

\begin{tabular}{lllll} 
Physical Functioning & ICU $(n=14)$ & & \multicolumn{2}{c}{ Surgery $\left(n=47^{*}\right)$} \\
& Mean & SD & Mean & SD \\
Before admission (PF1) & 38.24 & 16.08 & 41.92 & 3.52 \\
Following admission (PF2) & 28.57 & 13.16 & 40.11 & 12.59 \\
Change & 9.67 & 15.84 & 1.81 & 10.55 \\
$95 \%$ confidence interval & & 0.52 to 18.81 & & -1.29 to 4.91
\end{tabular}

Table 2. Physical functioning before and after hospital admission. *1 missing

Following discharge, within the ICU group no patients reported the ability to participate in vigorous activities without limitation; described in the survey as running, lifting heavy objects, and participating in strenuous sports, although four were able to participate in such activities pre-admission. Only one subject was able to take part in moderate activities without limitation; described as moving a table, pushing a vacuum cleaner, bowling or playing golf. Four ICU subjects were able to take part in such activities pre-admission.

It can be seen in Table 1 that the ICU group scored lower mean scores than the surgery group, this was investigated further by analysing subjects' response to their perception of their own health. The responses to question 2 (Compared to when you were admitted to hospital, how would you rate your health in general now?) are shown in Table 3. There were no statistically significant differences between the groups in terms of perceived health.

The relationship between length of stay and the subjects' scores in each of the categories was also analysed. However, no significant correlations were found.

\section{Summary of Results}

The results demonstrated the following:

* Subjects admitted to ICU for three nights or more had a significantly reduced level of physical function twelve weeks post discharge

* Twelve weeks following major general surgery subjects in this sample achieved the same level of physical function as they had prior to admission

* Social Functioning and the Emotional Role were affected more than other aspects of quality of life in this sample

* There was no relationship between hospital length of stay and the subjects' quality of life

\section{CONCLUSIONS}

\section{Limitations}

It is acknowledged that because the sample size for this study was relatively small and it was drawn from one geographical area of England, generalisability to a wider population is limited.

The main finding from this study was that quality of life following hospitalisation was reduced. For ICU survivors, physical function deteriorated significantly $(p=0.04)$, and for the whole sample social function, emotional and physical role were negatively affected.

On the basis of these findings it is suggested that rehabilitation programmes for ICU survivors should encompass physical exercise as well as emotional support. Further emotional support should also be offered to patients following general surgery.

These findings are also consistent with other research. As noted in the introduction, despite medical recovery, functional abilities following ICU admission do deteriorate. Psychological factors have not been investigated so widely, although it has been reported that a psychological approach to care complements physical

\begin{tabular}{|c|c|c|c|c|}
\hline \multirow[t]{2}{*}{ Perceived health } & \multicolumn{2}{|c|}{$\mathbf{I C U}$} & \multicolumn{2}{|c|}{ Surgery } \\
\hline & $\mathrm{n}$ & $\%$ & $\mathrm{n}$ & $\%$ \\
\hline Much better & 3 & 21 & 16 & 33 \\
\hline Somewhat better & 3 & 21 & 13 & 27 \\
\hline About the same & 4 & 29 & 13 & 27 \\
\hline Somewhat worse & 4 & 29 & 5 & 10 \\
\hline Much worse & - & - & 1 & 2 \\
\hline TOTAL & 14 & & 48 & \\
\hline
\end{tabular}

Table 3. Perceived health 
interventions during and following intensive care (Jones et al., 1998). However, psychosocial aspects have shown more rapid recovery than physical performance (Niskanen et al., 1999). There should be psychological input into any rehabilitation programme following intensive care or general surgery, the extent of which should be evaluated as it evolves.

The study also answered a call for evaluations of quality of life from critical care clinicians. A move away from short-term outcomes in order to evaluate the function of survivors is commonplace (Grady, 2001; Heyland and Kutsogiannis, 2000; Orlando, 2000; Curtis, 2002; Lewis, 2003). These results add to the evidence base in this evaluation of critical care therapies and will guide future practice in the local area of the study.

\section{ACKNOWLEDGEMENT}

The statistical assistance of Paul Finn, Medical Statistician, Teesside University, Middlebrough, UK is acknowledged.

\section{REFERENCES}

Curtis J R (2002). The long term outcomes of mechanical ventilation: what are they and how should they be used? Respiratory Care 47(4): 496-507.

Department of Health (2000) Comprehensive Critical Care: A Review of Adult Critical Care Services. London, HMSO.

Grady K (2001) Beyond morbidity and mortality: Quality of Life outcomes in critical care patients. Critical Care Medicine 29(9): 1844 1845.

Griffiths RD and Jones C (1999) Recovery from intensive Care. British Medical Journal 319(7207): 427-429.

Heyland DK, Hopman W, Coo H, Tranmer J, McColl M (2000) Long term health-related quality of life in survivors of sepsis. Short Form 36: A valid and reliable measure of health-related quality of life. Critical Care Medicine 28(11): 3599-3605.

Heyland DK and Kutsogiannis DJ (2000) Quality of Life following critical care: moving beyond survival. Intensive Care Medicine 26(9): 1172 1175.
Jones C, Humphris GM and Griffiths RD (1998) Psychological morbidity following critical illness - the rationale for care after intensive care. Clinical Intensive Care. 9(5): 199-205.

Jordan-Marsh M (2002) Advanced Practice: The SF-36 Quality of Life instrument: Updates and Strategies for Critical Care Research. Critical Care Nurse. 22(6): 35-43.

Lewis M (2003) Intensive care unit rehabilitation within United Kingdom. Physiotherapy. 89(9): 531-538.

McHugh LG, Milberg JA, Whitcomb ME, Schoene RB, Maunder RJ, Hudson LD (1994) Recovery of function in survivors of the Acute Respiratory Distress Syndrome. American Journal of Respiratory Critical Care Medicine 150: 90-94.

NHS Modernisation Agency (2003) Report in Developing Services: Critical Care Outreach 2003. London, Department of Health.

Nielsen D, Sellgren J and Ricksten S (1997) Quality of life after cardiac surgery complicated by multiple organ failure. Critical Care Medicine 25(1): 52-57.

Niskanen M, Ruokenen E, Takala J, Rissanen P, Kari A (1999) Quality of life after prolonged intensive care. Critical Care Medicine 27(6): 11321139.

Orlando R (2000) Quality of life in intensive care unit survivors: A place for outcomes research in critical care. Critical Care Medicine 28(11): 3755-3756.

Pettila V, Kaarlola A, Makelainen A (2000) Health-related quality of life of multiple organ dysfunction patients one year after intensive care. Intensive Care Medicine. 26(10): 1473-1479.

Ware JE, Bjorner JB, Kosinksi M (2000) Practical implications of item response theory and computerised adaptive testing. Medical Care 38(9 Suppl.): 1173-1182.

Ware JE, Kosinksi M and Dewsey (2000) How to score version 2 of the SF-36 health survey (standard and acute forms). USA, Medical Outcomes Trust and Quality Metric Inc.

Weinert CR, Gross CR, Kangas JR, Bury CL, Marinelli WA (1997) Health related quality of life after acute lung injury. American Journal of Respiratory Critical Care Medicine 156(4 Pt.1): 1120-1128. 